\title{
Interactive comment on "Microphysical Properties of Frozen Particles Inferred from Global Precipitation Measurement (GPM) Microwave Imager (GMI) Polarimetric Measurements” by Jie Gong and Dong L. Wu
}

\section{Anonymous Referee \#1}

Received and published: 19 October 2016

General comment:

This paper presents polarization observations of frozen particles from a spaceborne microwave imager. The topic is quite interesting and practical since polarization in the microwave spectral (especially $>150 \mathrm{GHz}$ ) is still not fully understood in the research community. The paper first analyzed the observations from GPM and found some features of PD-TB over land and ocean. It then introduced radiative transfer models to further discuss and explained the "bell" curve of PD-TB for ice cloud detection, and also presented the PD with/without melting layers. 
However, the paper is not so clear to me. Instead of discussing how the observed polarization signals can be used for further study and how this can further affect the accuracy of IWP retrievals in detail (which would be rather significant for future study), it only analyzed the observation results and only mentioned that $30 \%$ error will be caused if polarization is neglected both in the abstract and in the conclusion part. How exactly can the observation of PD improve the accuracy of IWP retrievals, how will PD be used? The results mentioned here are not convincing at all.

The structure of the paper is confusing. The authors described the observational data used in the study and analyzed the data, and then suddenly jumped to RT model description. Following the observational statistics in Section 3, another simple model was built up in Section 4. Basically, the paper was organized from data analysis to model description in Section 2, back to data statistics in Section 3, and then discussed with models again in Section 4. It seems to me that this is a bit chaotic.

At the end of Section 2.3, the authors introduced the concept of AR and defined it as the ratio of $\mathrm{V} / \mathrm{H}$ scattering coefficients. Then the authors mentioned in the following sections that this parameter AR is equivalent to what is mentioned in Davis et al. 2005. However, the AR in Davis at al. 2005 describes the shape of ice particles (the ratio of the long axis to short axis of spheroids), this is totally different from the AR defined in this paper (Section 2.3 and 4.1). The authors didn't discuss in detail (1) how this AR in the paper is affected by particle microphyscis at different frequencies (habit, orientation, size and so on); (2) Why AR is independent of height, considering the complex atmospheric conditions. To understand your conclusions in the following sections, the authors should also present what the simulated value of $A R$ is for different particles shapes/orientation/other microphysics.

I do believe the particle habit is related to the V/H scattering coefficients, but this is not the only factor. The orientation of ice particle, which was mentioned several times in the paper but not discussed here at all, is another important factor, which is related to AR defined in the paper.

Printer-friendly version

Discussion paper
Interactive

comment 
Section 4.1 introduced a simple model to explain the "bell" relation. However, as I mentioned above, I don't understand the AR values (Equation 4 should be $(T 1-T j)^{\star}($ tau_2htau_2v) ???). Why is AR in the range of 1.1-1.3? Why is Tj-T1 roughly constant? (P13, L4). To me, Tj depends on the location of ice cloud and T1 is related to the near surface temperature. Both $\mathrm{Tj}$ and $\mathrm{T} 1$ are varying with atmospheric conditions. And basically the assumption of constant Tj-T1 is not true as the authors mentioned in the paper.

Interactive

comment

The authors claimed that the PD-TB relationship is independent of channel frequency (P13, L11-15). This conclusion is based on the assumption of constant AR in the atmosphere at different frequencies. However, this assumption wasn't proved to be true in this paper. As shown Figure 2, 3, and 5, the maximum PD corresponds to different TB and depends on channel frequency, i.e., the PD-TB relationship changes. Also Figure 8 shows the dependence of PD-TB on AR at three different frequencies, which is not roughly 1.3 for all the frequencies at it showed.

P16, L1, How accurate is the BB flag? This has a significant effect on the conclusions. Section 4.3 didn't distinguish different precipitation types: whether rain or snow. When the authors can't distinguish the liquid/frozen precipitation, the results are still too rough. No BB could be snow precipitation as the authors mentioned. For snow precipitation the snow scattering is weak and $89 \mathrm{GHz}$ channels can still "see" the ocean surface. Thus the PD at $89 \mathrm{GHz}$ is strong. For rain with $\mathrm{BB}$, the near surface is screened by $\mathrm{BB}$ and rain, and the $\mathrm{BB}$ has polarization. Thus it could result in a higher PD as observed above. The mechanism is still too complicated and not clearly interpreted here.

Some of the figures in the paper are difficult to read. I suggest the authors to revise the figures to better understand the results. Eg. Figure 1, Left Bottom panel: you'd better use the same colorbar for comparison. It seems to me that at $89 \mathrm{GHz}$ PD is also up to $12-16 \mathrm{~K}$ and is comparable with the $166 \mathrm{GHZ} \mathrm{PD}$ values. Figure 2, the $\mathrm{y}$ axis range of the left and right panels are not the same and difficult to find the right value that described in the text. Figure 4, it is not easy to read it and please optimize the figure.

Printer-friendly version

Discussion paper 
Figure 10, The values of the color and contours are not described either in the figure or the figure caption.

Specific comment: 1.P1, L20. "increase slightly with latitude", How slight ? 2.P1, L25. the authors claimed that in deep convective cores, PD is reduced due to turbulence mixing. It is ambiguous, are there more ice or more liquid water? As the authors discussed in the text, attenuation by liquid water and water vapor lead to a decrease of PD. 3.P1, L34. references are missing here. 4.P1, L37. references are missing here. Please indicate which models you mentioned here. (better name one or two). 5.P2, L6. It is not appropriate to refer to Xie et al. 2015, better cite a general one. 6.P2, L9. I didn't find this reference in the bibliography (Xie, 2012) 7.P2, L15. inappropriate references (Miao et al 2003 and Xie and Miao 2011). It would be also good to mention the paper from Defer et al. 2014. SInce they also investigated polarization signals at $157 \mathrm{GHz}$ (Defer, E., V. Galligani, C. Prigent, and C. Jimenez, First observations of polarized scattering over ice clouds at close-to millimeter $\backslash$ frequencies $(157 \mathrm{GHz})$ with MADRAS on board the Megha-Tropiques mission, DOI : 10.1002/2014JD022353, J. Geophys. Res., 2014. ) 8.P2, L23. Davis et al. 2005 did observe polarized signals, but it is not significant as it was mentioned in the paper. 9.P6, L9, didn't find the reference in the bibliography Greenwald et al., 1997 10."habit" instead of "habitat" throughout the paper 11.P16, L22,"obsolete" or "oblate"?

Interactive comment on Atmos. Chem. Phys. Discuss., doi:10.5194/acp-2016-787, 2016. 